EPJ Web of Conferences 43, 01001 (2013)

DOI: $10.1051 /$ epjconf/20134301001

(C) Owned by the authors, published by EDP Sciences, 2013

\title{
Selected topics in the evolution of low-mass stars
}

\author{
M. Catelan ${ }^{1,2, a}$ \\ ${ }^{1}$ Departamento de Astronomía y Astrofísica, Pontificia Universidad Católica de Chile, \\ Av. Vicuña Mackenna 4860, 782-0436 Macul, Santiago, Chile \\ 2 The Milky Way Millennium Nucleus, Av. Vicuña Mackenna 4860, 782-0436 Macul, \\ Santiago, Chile
}

\begin{abstract}
Low-mass stars play a key role in many different areas of astrophysics. In this article, I provide a brief overview of the evolution of low-mass stars, and discuss some of the uncertainties and problems currently affecting low-mass stellar models. Emphasis is placed on the following topics: the solar abundance problem, mass loss on the red giant branch, and the level of helium enrichment associated to the multiple populations that are present in globular clusters.
\end{abstract}

\section{INTRODUCTION}

Low-mass stars are the most common type of stars in the Universe, and as such a proper understanding of their properties is of great astrophysical importance. The structure and evolution of low-mass stars has been the subject of several recent reviews [e.g., 19, 20, 22, 88, 93, and also the papers by M. Salaris and A. Weiss in these proceedings] - and therefore, in the present paper, I will only briefly discuss a few selected topics of current interest. I start by providing an overview of low-mass stellar evolution in $\$ 2$. A personal view of the current status of the so-called "solar abundance problem" is given in $\S 3$, and some recent developments in the computation of the mass-loss rates of red giants are described in $\S 4$. Some remarks on the helium content of the multiple populations that are present in globular clusters (GCs) are provided in $§ 5$. Finally, conclusions are given in $§ 6$.

\section{OVERVIEW}

The evolution of a "typical" (single) low-mass star accross the Hertzsprung-Russell diagram, from the zero-age main sequence (ZAMS) to the white dwarf (WD) cooling curve, is shown in Figure 1. All the main evolutionary phases are highlighted, including the main sequence (MS), subgiant branch (SGB), red giant branch (RGB), horizontal branch (HB), asymptotic giant branch (AGB), post-AGB, and WD phases. In addition, several key points in the evolution of the star are also labeled, namely: 1) ZAMS; 2) core $\mathrm{H}$ exhaustion/turn-off point; 3) first dredge-up episode; 4) RGB "bump"; 5) He core ignition under degenerate conditions (He "flash"); 6) zero-age HB (ZAHB); 7) core He exhaustion/commencement of the AGB phase. A zoomed-in version of this plot is provided in Figure 2; in this figure, the many gyrations or loops associated with thermonuclear flashes between the RGB tip and ZAHB are clearly seen, as are the loops associated with thermal pulses in AGB stars. Note that the star may cross the "classical" instability strip at several points in its life, including not only the HB (RR Lyrae stars) and AGB (type II Cepheids) phases, but also the pre-ZAHB phase ("RR Lyrae stars in disguise" [76]).

ae-mail: mcatelan@astro.puc.cl

This is an Open Access article distributed under the terms of the Creative Commons Attribution License 2.0, which permits unrestricted use, distribution, and reproduction in any medium, provided the original work is properly cited. 


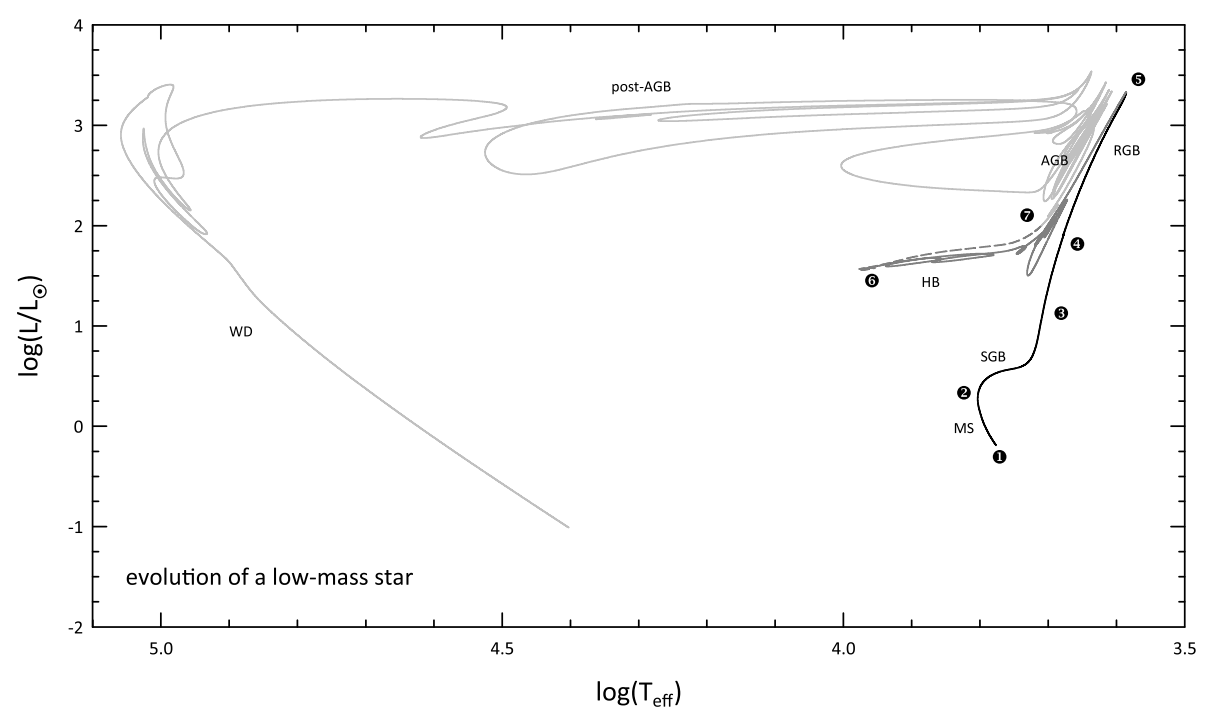

Figure 1. H-R diagram showing the evolution of a low-mass star with the following parameters: $Y=0.23$, $Z=0.0015, M=0.862 M_{\odot}$. The star loses a total of $\approx 0.26 M_{\odot}$ due to stellar winds during the RGB phase. The main evolutionary stages are labeled, and key stages in the star's life are numbered as follows: (1) ZAMS; (2) central H exhaustion and turn-off point; (3) first dredge-up episode; (4) RGB "bump"; (5) He ignition (RGB tip); (6) ZAHB; (7) central He exhaustion. Adapted from [22], based on computations by Brown et al. [15].

All these episodes in the life of a low-mass star (including, in addition, the pre-MS evolution) are extensively reviewed in [22, and references therein], and will thus not be repeated here.

\section{THE SOLAR ABUNDANCE PROBLEM}

\subsection{The problem}

Until about 2005, it was generally considered that evolutionary computations based on the solar metallicity $Z_{\odot}$ favored by N. Grevesse and co-authors [2, 41, 42] "yield solar models in good agreement with the data" [7, and references therein]. The perceived good agreement is illustrated by the solid line in Figure 3, which compares the predictions of the standard solar models computed by Bahcall et al. [8] for the sound speed $c(r)$ and the actual sound speeds that are inferred from helioseismology. The maximum deviation is of the order of $0.3 \%$. However, in 2005 new spectroscopic solar abundances were provided by Asplund et al. [5], based on impressive, highly sophisticated 3D hydrodynamical models of the solar atmosphere, which led to an important decrease in $Z_{\odot}$ (see Fig. 4). ${ }^{1}$ Again as shown in Figure 3, this led to a significantly worse agreement between the solar model computations and the helioseismological observations, with the maximum deviation now reaching a level of about $1.2 \%$.

This relatively recent development is commonly referred to as the "solar abundance problem." To be sure, and as can be seen in Figure 4, the $Z_{\odot}$ value went back up slightly with the extensive follow-up study by Asplund et al. [6], but this increase proved insufficient to restore the level of agreement that existed previously [e.g., 74].

\footnotetext{
1 This plot includes the $Z_{\odot}$ value estimated by [82] on the basis of the Holweger [47] abundance determinations for a few key elements, namely $\mathrm{C}, \mathrm{N}, \mathrm{O}, \mathrm{Ne}, \mathrm{Mg}, \mathrm{Si}, \mathrm{Fe}$, as reported on Table 1 of Guzik \& Mussack [43].
} 


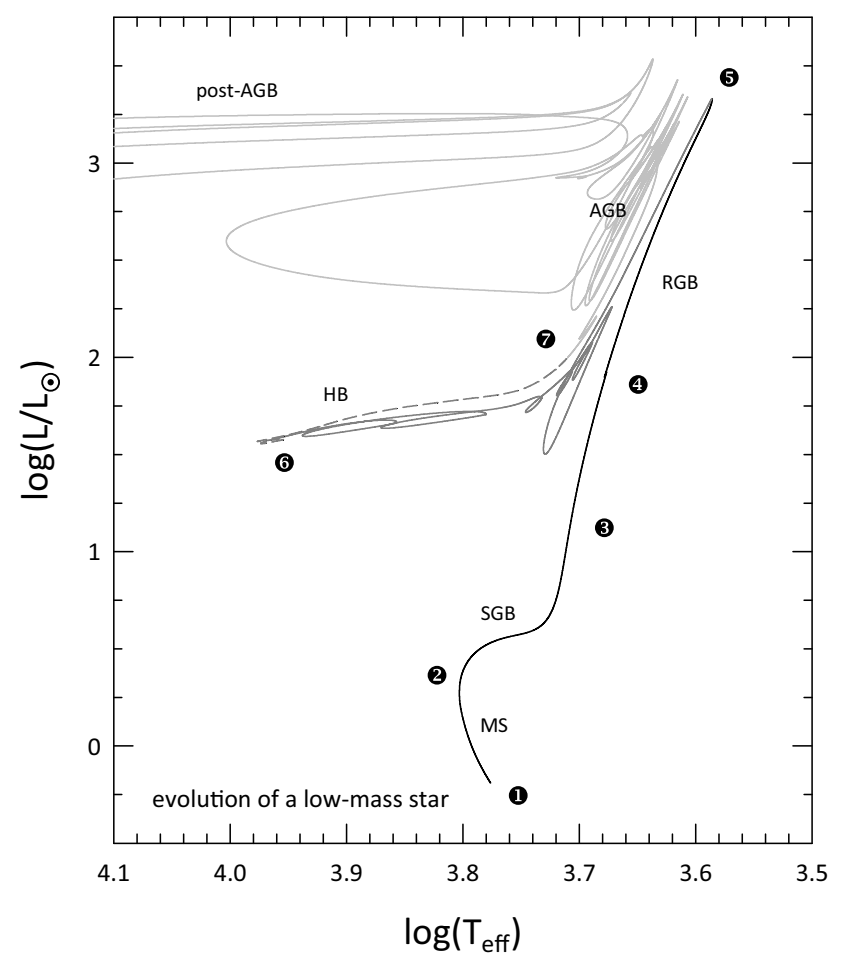

Figure 2. As in Figure 1, but zooming in on the main nuclear burning stages.

The solution to the problem is currently unknown, but several suggestions have been raised in the literature, none of which has proven entirely satisfactory. In what follows, a brief, and admittedly rather personal, overview is presented of some of the recent work that has been carried out in this area.

\subsection{Astrophysical perspective}

\subsubsection{Solar abundances: The present and the future}

Recently, and as also shown in Figure 4, the independent 3D hydrodynamic study by Caffau et al. [17], carried out using the $\mathrm{CO}^{5} \mathrm{BOLD}$ code, has brought a further increase in the solar metallicity, beyond that attained in Asplund et al. [6]. However, the Caffau et al. results have recently been subject to some criticism, particularly in regard to the selection of atomic line lists [40, Grevesse 2012, these proceedings]. Accordingly, a change in the $\mathrm{CO}^{5} \mathrm{BOLD} Z_{\odot}$ value cannot be excluded, at this point in time. However, it is unclear whether the change would bring values back down to the Asplund et al. levels. In particular, the controversy regarding the choice of line lists applies primarily to the carbon lines, and to a lesser extent to lines of other elements (Ludwig \& Caffau 2012, priv. comm.).

On the other hand, the possibility of yet another increase in $Z_{\odot}$ has been raised very recently by Fabbian et al. [33, 34]. Indeed, according to their recent 3D radiation-magnetohydrodynamic (MHD) computations, magnetic fields may play a very important role in deriving reliable abundances for the chemical elements in the Sun. Indeed, it has been known for several years now that the Sun has a substantial amount of "hidden" magnetic energy, in the form of tangled subsurface magnetic field lines, concentrated in intergranular lanes [54], with an average intensity of the order of 100-200 G [80], but including contributions also at the $\mathrm{kG}$ level [79]. This line of research should certainly be pursued further, but at present it remains unclear by how much (and even whether) such 3D MHD models will 


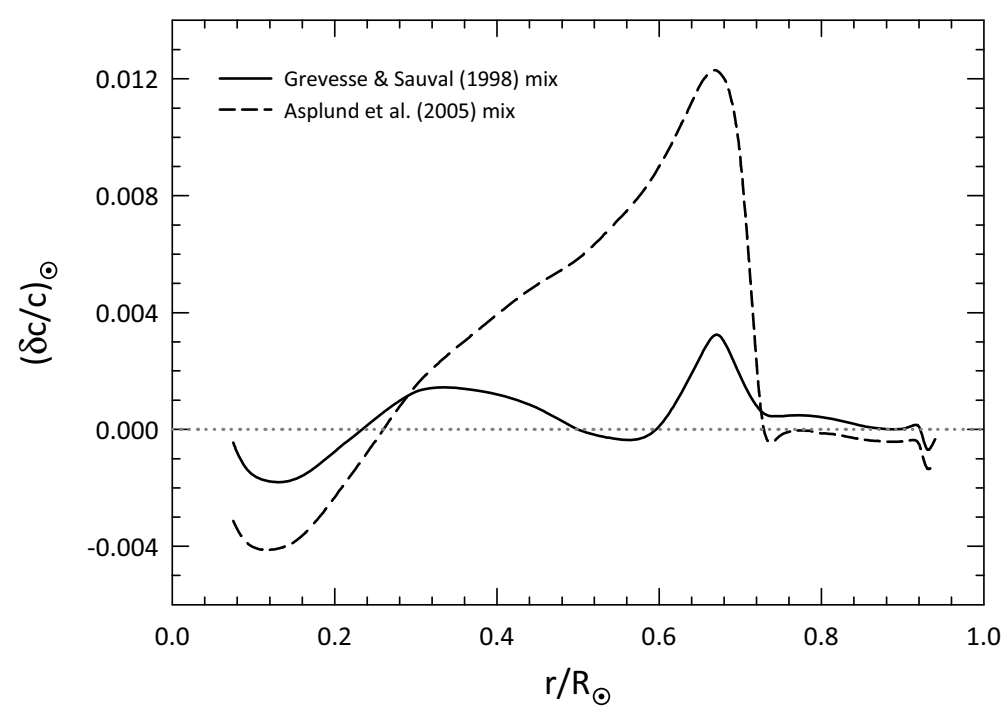

Figure 3. Illustration of the "solar abundance problem" circa 2005. The relative difference $(\delta c) / c$ between the sound speed as inferred from helioseismology and that predicted by the "standard solar model" is shown for two different choices of the solar heavy element mix: Grevesse \& Sauval [42, solid line] and Asplund et al. [5, dashed line $]$.

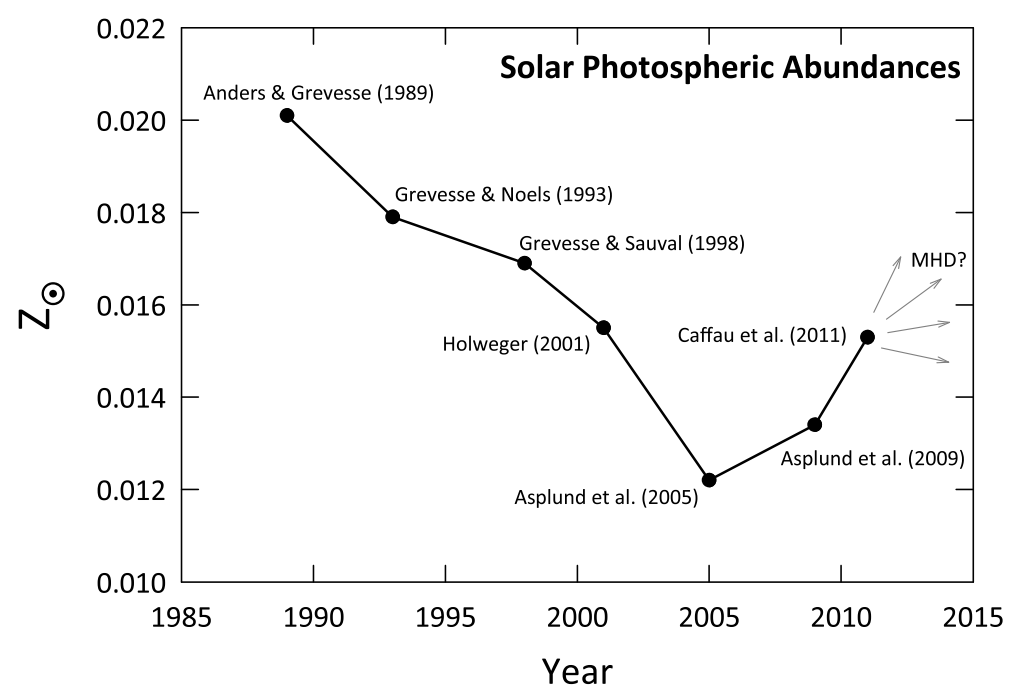

Figure 4. Evolution of the recommended solar metal abundance $Z_{\odot}$. The gray arrows point to the possible increase in $Z_{\odot}$ due to the inclusion of MHD effects, as recently suggested by Fabbian et al. [33, 34].

increase $Z_{\odot}$ beyond current levels. MHD effects are accordingly illustrated in Figure 4 as gray arrows with uncertain slope.

Recently, an increase in the Ne abundance, and/or in its contribution to the opacity, has been raised as a possible solution to the solar abundance problem [see 9, for a recent review]. However, the possibility of an increased $\mathrm{Ne}$ abundance has been disputed by Asplund et al. [6], and Lin et al. [55] show that increased $\mathrm{Ne}$ abundances actually worsen the agreement with the Sun, in the deeper part of the convection zone. 


\subsection{Physical perspective}

From a physical perspective, the situation immediately reminds one of that which was encountered a few decades ago in the field of stellar pulsation, with a persistent disagreement between computed and observed period ratios of double-mode (or "bump") Cepheids. Simon [77] realized that the problem could be solved by increasing radiative opacities by a factor of $2-3$, at temperatures $\sim 10^{5} \mathrm{~K}$. At the time, some authors considered that such a large increase in the opacity would be unrealistic [e.g., 56], but Simon's suggestions were eventually vindicated by the new opacity calculations by both the OPAL and OP teams [e.g., 48, 68, 72]. One may thus conjecture that further changes in the radiative opacities will occur in the future that will bring helioseismology back into agreement with a low $Z_{\odot}$.

Indeed, several authors have shown that an increase in the opacity by perhaps $10-30 \%$, in the temperature range $(3.5 \pm 1.5) \times 10^{6} \mathrm{~K}$, and particularly close to the base of the convective zone, plus a more modest increase close to the center, would serve to at least restore the previous level of agreement between solar models and helioseismology [e.g., 7, 9, 11, 27, 37, 61, 73, 91]. However, it is widely thought [e.g., 45, and references therein] that the level of uncertainty in current opacity calculations is at the level of $5 \%$ only [but see 83 , for a different point of view]. It thus remains to be seen whether such suggested opacity increases will materialize in the future, or whether alternative explanations for the solar abundance problem will have to be found. In this sense, Young \& Arnett [94] call attention to an alternative route towards increasing the "effective" opacity by means of internal gravity waves [64], which could provide another means of bringing solar models back in agreement with helioseismology.

While radiative opacities may be the most natural culprits, they are by no means the only possible ones; for instance, Basu [9, 10] points out that changes in opacity cannot be fully satisfactory without accompanying changes in the equation of state.

\subsubsection{Non-standard solar models}

Many authors have explored the possibility that standard solar models may be missing some fundamental physical processes, whose inclusion might help bring them into agreement with helioseismology. Among the possibilities that have been discussed in the literature, one may find the following: increased early mass loss [43]; accretion of low- $Z$ material from the protosolar nebula [43, 75]; convective overshooting [43]; realistic treatment of convection [4]; enhanced diffusion [e.g., 9, 44, and references therein]; rotation-induced mixing [16, 21, 82]; and combinations thereof [e.g., 84]. Among the more exotic solutions, one may find the conversion of photons to new light bosons in the solar photosphere [92], as well as alternative theories of gravity [18].

\subsection{An "Effective Solar Metallicity" for evolutionary databases}

At this point, it is worth reminding the reader that every set of evolutionary models currently in use by the astrophysical community at large is calibrated on the Sun. The procedure was pioneered by Demarque \& Larson [31] and Demarque \& Percy [32], and requires that the present-day luminosity (or effective temperature) and radius of the Sun are matched with the present-day (i.e., at an age of $4.57 \mathrm{Gyr}$ ), precisely measured solar values. In this way, one obtains the solar helium abundance $Y_{\odot}$ and the mixing length parameter $\alpha_{\mathrm{MLT}}$ of mixing length theory, for an assumed solar metallicity: for instance, in the original work by Demarque \& Larson, a solar metallicity $Z_{\odot}=0.03$ was adopted. This procedure has remained essentially unchanged over the past (almost) 50 years, and so present-day stellar evolution calculations, and the extensive sets of evolutionary tracks, isochrones, and luminosity functions derived therefrom, and applied to studies of resolved and unresolved, Galactic and extragalactic, stellar populations, are still calibrated in much the same way - the main difference being that the adopted value of the solar metallicity has changed with time (see Fig. 4). Note that the very same procedure is always adopted, irrespective of the degree of physical refinements that are included or lacking in any 


\section{EPJ Web of Conferences}

set of models - for instance, gravitational settling, radiative acceleration, turbulent mixing, rotation, magnetic fields, treatment of convection beyond the extremely simplified MLT, etc.

It is perhaps advisable, at this point in time, to recognize the limitations both of our input physics and models, and to calibrate these models using an additional quantity that was not available to the pioneers in the field, but which is now very precisely measured - namely, the internal sound speed profile $c(r)$, as obtained from helioseismology [e.g., 8, 12]. Solar neutrino rates can also be included in such a "beefed up" calibration process, thus providing yet another route to help further constrain the innermost layers of low-mass stellar models [e.g., 3, 46, 75, 81, 91]. Indeed, one is now in a priviledged position, from the standpoint of the available empirical data, to require of one's solar models a closer match not only of the Sun's luminosity and radius at an age of $4.57 \mathrm{Gyr}$, but also of $c(r)$ - and especially the depth of the convection zone - in addition to the observed neutrino rates. To achieve this, an "effective solar metallicity" $Z_{\odot \text {,eff }}$ may accordingly be defined in such a way that an optimum match of the present-day, precisely measured properties of the Sun - namely, its luminosity, radius, $c(r)$, and neutrino rates yields $Y_{\odot}, \alpha_{\mathrm{MLT}}$, and $Z_{\odot, \text { eff }}$.

Ideally, in the presence of perfect physical input, perfect spectroscopic abundances, and perfect numerical models, $Z_{\odot, \text { eff }}$ will be identical to the spectroscopically measured photospheric solar

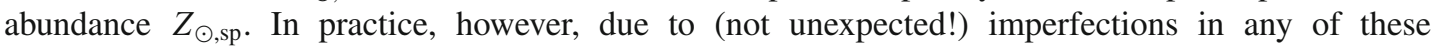
ingredients, calibration of the solar model will frequently give $Z_{\odot \text {,eff }} \neq Z_{\odot \text {,sp }}$.

Whatever the explanation of the solar abundance problem, it is clear that solar models computed using the Asplund et al. [5, 6] or Caffau et al. [17] solar abundances fail to correctly predict the sound speed profile that is inferred from helioseismology. The fact that models computed using higher solar metallicities, as had previously been favored in the literature [e.g., 2, 41, 42], provide a better match to the helioseismological observations strongly suggests that either the new solar metallicities are too low, or/and the physical ingredients that are used in the computation of stellar models, and especially those that depend strongly on the metallicity (such as radiative opacities), are somehow in error. Be as it may, present-day standard solar models computed with low metallicities cannot be seen as properly describing the inner structures of low-mass stars, even if the low solar metallicity is correct. Evolutionary models (and the isochrones derived therefrom) calibrated on the Sun in the more robust way just described, on the other hand, though certainly not perfect, should somehow more realistically describe the evolutionary paths of such stars, since they - by definition - more successfully reproduce the internal structure of the Sun.

To close, we note that van Saders \& Pinsonneault [89] recently suggested that stellar metallicities derived in a way much as has just been described, based on helio- and asteroseismology, could be used to define a new, absolute stellar abundance scale. References to related prior work can be found in the reviews by Mussack \& Gough [62], Basu [9, 10], and Gough [36, 37], among others.

\section{MASS LOSS IN RED GIANTS}

Whether in their first or second ascent of the red giant branch, low-mass stars are expected to lose large amounts of mass as they evolve. In the calculation of evolutionary tracks, it is still a common procedure to adopt the Reimers $[65,66]$ analytical expression to quantitatively evaluate the corresponding mass loss rates. However, many other formulations have been proposed in the more recent literature, and the differences in predicted quantities, such as the integrated RGB mass loss and its dependence on metallicity, can be quite substantial, with a strong impact on the predicted RGB progeny [e.g., 24, 53, and references therein]. It is thus very important to establish proper recipes for the mass loss rates in low-mass red giants, guided by the latest available empirical data.

In this sense, it is now well established that the Reimers expression does not properly describe the behavior of mass loss in red giants [e.g., 28, 69, 70], and should thus not be used in state-of-the-art evolutionary calculations. 
Fortunately, several improved formulations have become available recently. In particular, Schröder \& Cuntz [69] proposed the following generalized formulation of the original Reimers expression (their eq. 4, as modified by [87] for further flexibility in evolutionary computations):

$$
\dot{M}=8 \times 10^{-14} \eta_{\mathrm{SC}} \frac{L_{\star} R_{\star}}{M_{\star}}\left(\frac{T_{\mathrm{eff}}}{4000 \mathrm{~K}}\right)^{3.5}\left(1+\frac{g_{\odot}}{4300 g_{\star}}\right) \quad\left(M_{\odot} \mathrm{yr}^{-1}\right)
$$

where $L_{\star}, R_{\star}, M_{\star}$, are the stellar luminosity, radius, and mass, respectively, given in solar units; $T_{\text {eff }}$ is the star's effective temperature, in $\mathrm{K} ; g_{\star}$ and $g_{\odot}$ are the stellar and solar surface gravities, respectively; and $\eta_{\mathrm{SC}}$ is a dimensionless coefficient of order 1. In this way, as shown by Schröder \& Cuntz [69], one is able to take into account both the mechanical energy flux that is added to the outer layers of the star and the dependence on chromospheric height, which are two key ingredients that were not satisfactorily included in the original Reimers formulation. The numerical factor $8 \times 10^{-14}$ was adjusted by Schröder \& Cuntz [69] to provide an optimum fit to HB stars in two GCs, namely M5 (NGC 5904) and NGC 5927. Then, assuming $\eta_{\mathrm{SC}}=1$, Schröder \& Cuntz [70] have shown that, unlike with the Reimers formula, one is able to obtain good agreement, within the error bars, with the empirical data for well-studied red giants and supergiants.

Very recently, a predictive theoretical model for the mass-loss rates of cool stars has been proposed by Cranmer \& Saar [28] which seems to provide an even better fit to the empirical data [see also 1]. The model follows the flux of MHD turbulence from subsurface convection zones to its eventual dissipation and escape through open magnetic flux tubes. The main drawback, as far as applications to the calculation of stellar evolutionary tracks is concerned, is the fact that their formulation does not reduce to a simple analytical formula - no doubt the main reason for the continued popularity of the Reimers $[65,66]$ expression, its well-established deficiencies notwithstanding. Instead, information on each individual star's magnetic fields and activity is a priori required.

Given the seemingly universal character of the quiet-Sun chromosphere among inactive stars, and thus the established concrete possibility of guiding mechanical energy toward the acceleration zone of cool stellar winds along flux tubes [71], further exploration of the Cranmer \& Saar [28] formulation in actual evolutionary calculations would prove of great interest, and is thus strongly encouraged. In particular, magnetic field variations may play a relevant role in the context of the second-parameter problem of HB morphology [see 23, for a review and references], possibly being responsible, for instance, for the existence of star-to-star variations in total mass along the HBs of individual GCs [e.g., 85], whose origin has long remained a mystery. In this sense, it would be especially interesting if the $\eta_{\text {SC }}$ parameter in eq. (1) could be calibrated in terms of the magnetic field-related quantities that appear in the Cranmer \& Saar models.

\section{MULTIPLE POPULATIONS IN GLOBULAR CLUSTERS}

It is now quite well established that GCs are not the simple stellar systems that they were once thought to be. In particular, evidence for the presence of multiple populations, interpreted as multiple star formation episodes with different degrees of associated chemical enrichment, has been obtained on the basis of both photometric and spectroscopic techniques [see, e.g., 14, 30, 38, 67, 86, for recent reviews and extensive references].

One of the most exciting aspects brought about by these recent developments is the perceived possibility of finding a physically well-motivated solution to the long-standing second-parameter problem. Indeed, while it has long been suspected that the abundance anomalies that are observed in GC red giants could be associated with the morphology of the HB [e.g., 25, 63], it is only recently that large enough observational databases have been amassed that allow more reliable tests to be carried out. 


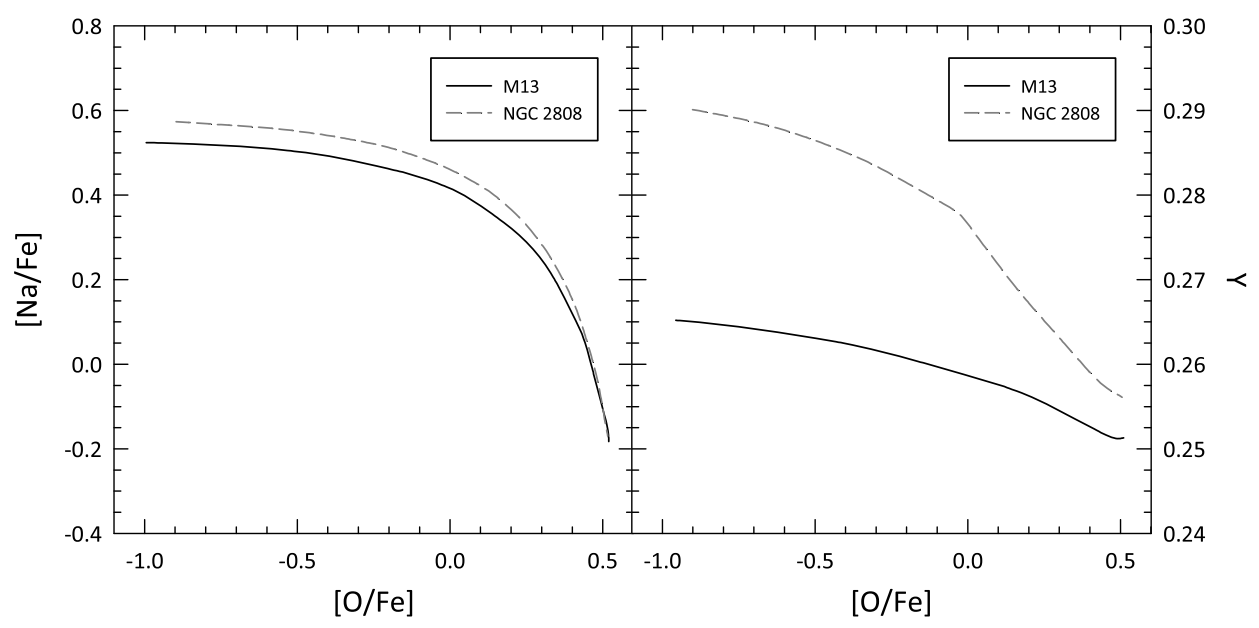

Figure 5. Chemical evolution models for two GCs, namely M13 (NGC 6205, solid lines) and NGC 2808 (dashed lines). Left: the $[\mathrm{Na} / \mathrm{Fe}]$ vs. $[\mathrm{O} / \mathrm{Fe}]$ anticorrelation. Right: predicted correlation between helium abundance $Y$ and [O/Fe]. Note the different $Y$ levels that are predicted for the same [O/Fe]. Adapted from [57].

In this context, Gratton et al. [39] argue that such abundance anomalies appear to be less important than only metallicity and age, as far as the second-parameter phenomenon of HB morphology goes.

According to most authors [e.g., 29], the main driver of blue HB morphologies, in the face of abundance variations in (mainly) $\mathrm{O}, \mathrm{Na}, \mathrm{Mg}$, and $\mathrm{Al}$, is the associated level of $\mathrm{He}$ enhancement that is brought about in the course of the operation of the $\mathrm{Ne}-\mathrm{Na}$ and $\mathrm{Mg}$-Al proton-capture cycles, which give rise, for instance, to the well-known O-Na anticorrelation. Uncertainties in the relevant nuclear reaction rates can be quite large, reaching orders of magnitude in some cases [e.g., 49-51]. In fact, the precise level of He enhancement that may be associated with a given degree of $\mathrm{O}$ depletion/ $\mathrm{Na}$ (and $\mathrm{Al}$ ) enhancement is not known a priori. As an example, in Figure 5 (left panel) the predicted correlation between $[\mathrm{Na} / \mathrm{Fe}]$ and $[\mathrm{O} / \mathrm{Fe}]$ [adapted from 57] is shown for two well-known GCs, namely M13 (NGC 6205) and NGC 2808, both of which harbor large numbers of extreme HB stars. The right panel in the same figure shows the associated trend between the helium abundance $Y$ and $[\mathrm{O} / \mathrm{Fe}]$. It is especially noteworthy that extremely low levels of $[\mathrm{O} / \mathrm{Fe}]$ may be reached, with only a minor level of associated He enrichment. In addition, closely the same $[\mathrm{O} / \mathrm{Fe}]$ and $[\mathrm{Na} / \mathrm{Fe}]$ ratios may imply widely different $\mathrm{He}$ enrichment levels. Last but not least, note also that some of the variation in the observed abundance ratios may be evolutionary in nature (i.e., reflecting mixing processes operating in the interiors of the red giants), as recently demonstrated by [52]. It is important to keep these points in mind, before arriving at conclusions regarding the level of He enrichment that may be present in a given population, based solely on measurements of light-element abundance ratios.

As a case in point, consider the GCs M3 (NGC 5272) and M4 (NGC 6121). As shown by Sneden et al. [78], M3 possesses a large fraction of "super-oxygen-poor" stars, by which we mean stars with $[\mathrm{O} / \mathrm{Fe}]<0$. Based on this datum alone, one might be tempted to suspect that $\mathrm{M} 3$ possesses a large number of He-enhanced stars. Yet, as shown by Catelan et al. [26] on the basis of high-precision Strömgren photometry, the level of He enhancement for the vast majority of M3 stars seems to satisfy $\Delta Y<0.01$. M4, on the other hand, according to the spectroscopic measurements by Marino et al. $[58,59]$, seems to be basically devoid of super-O-poor stars - and yet, according to Villanova et al. [90], blue HB stars in this cluster somehow present a fairly high level of He enhancement, of order $\Delta Y \approx 0.02-0.04$. It remains to be established why it is that $\mathrm{M} 3$, with its significant levels of oxygen depletion, was somehow unable to produce HB stars with comparable levels of He enhancement as claimed for M4, whose stars do not reach similarly low [O/Fe] ratios as seen in M3. 


\section{Ageing Low Mass Stars: From Red Giants to White Dwarfs}

\section{CONCLUSIONS}

Several issues remain open in our knowledge of the evolutionary properties of low-mass stars. In this paper, a few topics that have been the subject of considerable debate in the recent literature have been addressed, namely the solar abundance problem, the mass loss rates in red giants, and the level of He enhancement in the different populations that are present within individual GCs. While considerable progress in all these areas has been made recently, more work will clearly be required before we are in a position to fully predict the evolutionary history of a low-mass star.

I would like to thank E. Caffau, H.-G. Ludwig, A. V. Sweigart, A. A. R. Valcarce, and N. Viaux, for several useful discussions and information. This work is supported by the Chilean Ministry for the Economy, Development, and Tourism's Programa Iniciativa Científica Milenio through grant P07-021-F, awarded to The Milky Way Millennium Nucleus; by Proyecto Fondecyt Regular \#1110326; by the BASAL Center for Astrophysics and Associated Technologies (PFB-06); and by Proyecto Anillo ACT-86.

\section{References}

[1] Airapetian, V., Carpenter, K.G., \& Ofman, L. 2010, ApJ, 723, 1210

[2] Anders, E., \& Grevesse, N. 1989, Geoch. Cosmoch. Acta, 53, 197

[3] Antonelli, V., Miramonti, L., Peña-Garay, C., \& Serenelli, A. 2012, preprint (arXiv:1208.1356)

[4] Arnett, D., Meakin, C., \& Young, P.A. 2010, ApJ, 710, 1619

[5] Asplund, M., Grevesse, N., \& Sauval, A.J. 2005, in Cosmic Abundances as Records of Stellar Evolution and Nucleosynthesis, ed. T.G. Barnes III \& F.N. Bash (San Francisco: ASP), ASP Conf. Ser., 336, 25

[6] Asplund, M., Grevesse, N., Sauval, A.J., \& Scott, P. 2009, ARA\&A, 47, 481

[7] Bahcall, J.N., Basu, S., Pinsonneault, M., \& Serenelli, A.M. 2005, ApJ, 618, 1049

[8] Bahcall, J.N., Serenelli, A.M., \& Basu, S. 2006, ApJS, 165, 400

[9] Basu, S. 2009, in Solar-Stellar Dynamos as Revealed by Helio- and Asteroseismology, ed. M. Dikpati et al. (San Francisco: ASP), ASP Conf. Ser., 416, 193

[10] Basu, S. 2010, Ap\&SS, 328, 43

[11] Basu, S., \& Antia, H.M. 2004, ApJ, 606, L85

[12] Basu, S., Pinsonneault, M.H., \& Bahcall, J.N. 2000, ApJ, 529, 1084

[13] Beeck, B., Collet, R., Steffen, M., et al. 2012, A\&A, 539, A121

[14] Bekki, K. 2011, MNRAS, 412, 2241

[15] Brown, T.M., Sweigart, A.V., Lanz, T., Landsman, W.B., \& Hubeny, I. 2001, ApJ, 562, 368

[16] Brun, A.S., Turck-Chièze, S., \& Zahn, J.P. 1999, ApJ, 525, 1032

[17] Caffau, E., Ludwig, H.-G., Steffen, M., Freytag, B., \& Bonifacio, P. 2011, Sol. Phys., 268, 255

[18] Casanellas, J., Pani, P., Lopes, I., \& Cardoso, V. 2012, ApJ, 745, 15

[19] Cassisi, S. 2011, MSAIt, in press (arXiv:1111.6464)

[20] Cassisi, S. 2012, Ap\&SS Proc., 2, 57

[21] Castro, M., Vauclair, S., \& Richard, O. 2007, A\&A, 463, 755

[22] Catelan, M. 2007, in Graduate School in Astronomy: XI Special Courses at the National Observatory of Rio de Janeiro (XI CCE), ed. F. Roig et al., AIP Conf. Proc., 930, 39

[23] Catelan, M. 2009a, Ap\&SS, 320, 261

[24] Catelan, M. 2009b, in New Quests in Stellar Astrophysics. II. Ultraviolet Properties of Evolved Stellar Populations, ed. M. Chávez et al. (Berlin: Springer), 175

[25] Catelan, M., \& de Freitas Pacheco, J.A. 1995, A\&A, 297, 345 
[26] Catelan, M., Grundahl, F., Sweigart, A.V., Valcarce, A.A.R., \& Cortés, C. 2009, ApJ, 695, L97

[27] Christensen-Dalsgaard, J., di Mauro, M.P., Houdek, G., \& Pijpers, F. 2009, A\&A, 494, 205

[28] Cranmer, S.R., \& Saar, S.H. 2011, ApJ, 741, 54

[29] D’Antona, F., \& Caloi, V. 2008, MNRAS, 390, 693

[30] Decressin, T. 2011, in Active OB Stars: Structure, Evolution, Mass Loss, and Critical Limits, IAU Symposium 272, ed. C. Neiner, G. Wade, G. Meynet, \& G. Peters (Cambridge: Cambridge Univ. Press), 227

[31] Demarque, P.R., \& Larson, R.B. 1964, ApJ, 140, 544

[32] Demarque, P.R., \& Percy, J.R. 1964, ApJ, 140, 541

[33] Fabbian, D., Khomenko, E., Moreno-Insertis, F., \& Nordlund, Å. 2010, ApJ, 724, 1536

[34] Fabbian, D., Moreno-Insertis, F., Khomenko, E., \& Nordlund, Å. 2012, A\&A, 548, 35

[35] Freytag, B., Steffen, M., Ludwig, H.-G., Wedemeyer-Böhm, S., Schaffenberger, W., \& Steiner, O. 2012, J. Comp. Phys., 231, 919

[36] Gough, D. 2011, in Astrophysical Dynamics: From Stars to Galaxies, IAU Symposium 271, ed. N.H. Brummell, A.S. Brun, M.S. Miesch, \& Y. Ponty (Cambridge: Cambridge Univ. Press), 3

[37] Gough, D.O. 2012, in Progress in Solar/Stellar Physics with Helio- and Asteroseismology, Proc. 65th Fujihara Seminar, ASP Conf. Ser., 462, 429

[38] Gratton, R.G., Carretta, E., \& Bragaglia, A. 2012, A\&A Rev., 20, 50

[39] Gratton, R.G., Carretta, E., Bragaglia, A., Lucatello, S., \& D’Orazi, V. 2010, A\&A, 517, A81

[40] Grevesse, N., Asplund, M., Sauval, A.J., \& Scott, P. 2011, Can. J. Phys., 89, 327

[41] Grevesse, N., \& Noels, A. 1993, in Origin and Evolution of the Elements, ed. N. Prantzos, E. Vangioni-Flam, \& M. Cassé (Cambridge: Cambridge Univ. Press), 15

[42] Grevesse, N., \& Sauval, A.J. 1998, Space Sc. Rev., 85, 161

[43] Guzik, J.A., \& Mussack, K. 2010, ApJ, 713, 1108

[44] Guzik, J.A., Watson, L.S., \& Cox, A.N. 2005, ApJ, 627, 1049

[45] Guzik, J.A., Watson, L.S., \& Cox, A.N. 2006, MSAIt, 77, 389

[46] Haxton, W.C., Hamish Robertson, R.G., \& Serenelli, A.M. 2012, preprint (arXiv:1208.5723)

[47] Holweger, H. 2001, in Solar and Galactic Composition, ed. R.F. Wimmer-Schweingruber, AIP Conf. Proc., 598, 23

[48] Iglesias, C.A., Rogers, F.J., \& Wilson, B. G. 1987, ApJ, 322, L45

[49] Iliadis, C., Champagne, A., Chieffi, A., \& Limongi, M. 2011, ApJS, 193, 16

[50] Iliadis, C., Longland, R., Champagne, A.E., \& Coc, A. 2010a, Nucl. Phys. A, 841, 323

[51] Iliadis, C., Longland, R., Champagne, A.E., Coc, A., \& Fitzgerald, R. 2010b, Nucl. Phys. A, 841, 31

[52] Johnson, C.I., \& Pilachowski, C.A. 2012, ApJ, 754, L38

[53] Kalirai, J.S., Saul Davis, D., Richer, H.B., Bergeron, P., Catelan, M., Hansen, B.M.S., \& Rich, R.M. 2009, ApJ, 705, 408

[54] Kitiashvili, I.N., Abramenko, V.I., Goode, P.R., Kosovichev, A.G., Lele, S.K., Mansour, N.N., Wray, A. A., \& Yurchyshyn, V.B. 2012, Phys. Scripta, in press (arXiv:1206.5300)

[55] Lin, C.-H., Antia, H.M., \& Basu, S. 2007, ApJ, 668, 603

[56] Magee, N.H., Jr., Merts, A.L., \& Huebner, W.F. 1984, ApJ, 283, 264

[57] Marcolini, A., Gibson, B.K., Karakas, A.I., \& Sánchez-Blázquez, P. 2009, MNRAS, 395, 719

[58] Marino, A.F., Villanova, S., Milone, A.P., Piotto, G., Lind, K., Geisler, D., \& Stetson, P. B. 2011, ApJ, 730, L16

[59] Marino, A.F., Villanova, S., Piotto, G., Milone, A.P., Momany, Y., Bedin, L.R., \& Medling, A.M. 2008, A\&A, 490, 625

[60] McDonald, I., \& van Loon, J.T. 2007, A\&A, 476, 1261

[61] Montalbán, J., Miglio, A., Noels, A., Grevesse, N., \& di Mauro, M.P. 2004, in Proc. SOHO 14/GONG 2004 Workshop, Helio- and Asteroseismology: Towards a Golden Future, 574 
[62] Mussack, K., \& Gough, D. 2009, in Solar-Stellar Dynamos as Revealed by Helio- and Asteroseismology, ed. M. Dikpati et al. (San Francisco: ASP), ASP Conf. Ser., 416, 203

[63] Norris, J. 1981, ApJ, 248, 177

[64] Press, W.H., \& Rybicki, G.B. 1981, ApJ, 248, 751

[65] Reimers, D. 1975a, in Problems in Stellar Atmospheres and Envelopes, ed. B. Baschek, W.H. Kegel, \& G. Traving (Berlin: Springer), 229

[66] Reimers, D. 1975b, Mem. Soc. R. Liège 6 Sér., 8, 369

[67] Renzini, A. 2008, MNRAS, 391, 354

[68] Rogers, F.J., \& Iglesias, C.A. 1992, ApJS, 79, 507

[69] Schröder, K.-P., \& Cuntz, M. 2005, ApJ, 630, L73

[70] Schröder, K.-P., \& Cuntz, M. 2007, A\&A, 465, 593

[71] Schröder, K.-P., Mittag, M., Pérez Martínez, M.I., Cuntz, M., \& Schmitt, J.H.M.M. 2012, A\&A, 540, A130

[72] Seaton, M.J., Yan, Y., Mihalas, D., \& Pradhan, A.K. 1994, MNRAS, 266, 805

[73] Serenelli, A.M. 2010, Ap\&SS, 328, 13

[74] Serenelli, A.M., Basu, S., Ferguson, J.W., \& Asplund, M. 2009, ApJ, 705, L123

[75] Serenelli, A.M., Haxton, W.C., \& Peña-Garay, C. 2011, ApJ, 743, 24

[76] Silva Aguirre, V., Catelan, M., Weiss, A., \& Valcarce, A.A.R. 2008, A\&A, 489, 1201

[77] Simon, N.R. 1982, ApJ, 260, L87

[78] Sneden, C., Kraft, R.P., Guhathakurta, P., Peterson, R.C., \& Fulbright, J.P. 2004, AJ, 127, 2162

[79] Stenflo, J.O. 2012, A\&A, 541, A17

[80] Trujillo Bueno, J., Shchukina, N., \& Asensio Ramos, A. 2004, Nature, 430, 326

[81] Turck-Chièze, S., \& Couvidat, S. 2011, Rep. Progr. Phys., 74, 086901

[82] Turck-Chièze, S., Couvidat, S., Piau, L., Ferguson, J., Lambert, P., Ballot, J., García, R.A., Nghiem, P. 2004, Phys. Rev. Lett., 93, 211102

[83] Turck-Chièze, S., Loisel, G., Gilles, D., et al. 2011a, J. Phys. Conf. Ser., 271, 012035

[84] Turck-Chièze, S., Piau, L., \& Couvidat, S. 2011b, ApJ, 731, L29

[85] Valcarce, A.A.R., \& Catelan, M. 2008, A\&A, 487, 185

[86] Valcarce, A.A.R., \& Catelan, M. 2011, A\&A, 533, A120

[87] Valcarce, A.A.R., Catelan, M., \& Sweigart, A.V. 2012, A\&A, 547, A5

[88] Valle, G., Dell'Omodarme, M., Prada Moroni, P.G., \& Degl'Innocenti, S. 2013, A\&A, 549, 50

[89] van Saders, J.L., \& Pinsonneault, M.H. 2012, ApJ, 746, 16

[90] Villanova, S., Geisler, D., Piotto, G., \& Gratton, R. G. 2012, ApJ, 748, 62

[91] Villante, F.L. 2010, ApJ, 724, 98

[92] Vincent, A.C., Scott, P., \& Trampedach, R. 2012, preprint (arXiv:1206.4315)

[93] Weiss, A. 2006, MSAIt, 77, 43

[94] Young, P.A., \& Arnett, D. 2005, ApJ, 618, 908 